\title{
弥拜霉素类似物的合成、表征和杀虫活性研究
}

\author{
徐小军 ${ }^{a, b}$ 赖声洪 ${ }^{a}$ 季明华 $c$ 朱国念 ${ }^{a}$ 赵金浩 $*, a, b$ \\ $\left({ }^{a}\right.$ 浙江大学农药与环境毒理研究所 农业部作物病虫分子生物学重点开放实验室 杭州 310029) \\ ( ${ }^{b}$ 浙江工业大学药学院 杭州 310014) \\ ( ${ }^{c}$ 浙江理工大学科学研究所 杭州 310032)
}

\begin{abstract}
摘要 以弥拜霉素类似物依维菌素为原料, 根据类同合成法和亚结构连接法原理, 对依维菌素进行脱糖, 再与相应的 酰氯进行酯化、肟化反应制得两个系列弥拜需素类似物化合物 $4 \mathbf{I a} \sim \mathbf{5 I I d}$, 所有目标化合物都通过核磁共振氢谱、高分 辨质谱的确认，并分别对朱砂叶螨(Tetranychus cinnabarinus)、南方粘虫(Mythimna sepatara)和寔豆蚜(Aphis fabae)进行 室内杀虫活性测定，结果表明所有衍生物均表现出不同程度的杀虫活性，其中化合物 4IIa 和 4IIb 对粘虫和蚜虫表现出 很高的杀虫活性.
\end{abstract}

关键词＼cjkstart弥拜霉素类似物；合成；杀虫活性

\section{Synthesis, Characterization and Insecticidal Activity of Milbemycin Analogues}

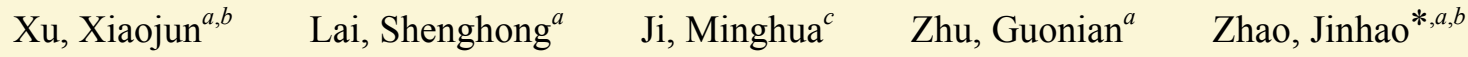 \\ ( ${ }^{a}$ Ministry of Agriculture Key Laboratory of Molecular Biology of Crop Pathogens and Insects, Institute of Pesticide and \\ Environmental Toxicology, Zhejiang University, Hangzhou 310029) \\ ( ${ }^{b}$ College of Pharmacy, Zhejiang University of Technology, Hangzhou 310014) \\ ( ${ }^{c}$ Instituent of Science, Zhejiang Sci-Tech University, Hangzhou 310032)
}

\begin{abstract}
Two series of milbemycin analogues containing ester and methoxy oxime groups were designed according to ana$\log$ synthesis and sub-structure ligation from deglycosyl ivermectin, which is the analogue of milbemycin. The analogues reaction was carried out with the corresponding aromatic chloride, and the structures of the target compounds were confirmed by ${ }^{1} \mathrm{H}$ NMR and high resolution mass spectra (HRMS). The insecticidal activities of 18 analogues against Tetranychus cinnabarinus, Mythimna sepatara and Aphis fabae were tested. The results showed that all analogues had different degree of insecticidal activity, especially compounds 4IIa and 4IIb showed the highest insecticidal activities against Mythimna sepatara and Aphis fabae.
\end{abstract}

Keywords milbemycin analogues; synthesis; biological activity

弥拜霉素(Milbemycins)为十六元大环内酯抗生素 类杀虫、杀螨、驱虫剂，由 Sankyo 公司和美国 MERCK 公司于 1975 年分别从链霸菌 (Streptomyces hygroscopius) 发酵产物中分离而得 ${ }^{[1,2]}$. 该类化合物具有结构 新颖、作用机制独特、生物活性广谱且高效、有效剂量 低、易于降解、安全性高等特点, 而吸引了许多科学家 进行研究. 自弥拜霉素 A3/A4 第一次成功商品化以来, 弥拜霉素类作为杀虫剂得到了快速发展 ${ }^{[3]}$, 尤其是 Takeshiba 等 ${ }^{[4]}$ 研发的 Lepimectin [13-( $\alpha$-甲氧亚氨基)苯
乙酰基弥拜霉素，结构式见 Scheme 1]的成功上市，更 体现了研究弥拜霉素类杀虫、驱虫剂的美好前景.

然而在国内，目前为止弥拜霉素类化合物未能作为 杀螨剂、杀虫剂而得到广泛应用，原因在于由 Sankyo 公司提供的弥拜霉素原料药昂贵. 为多渠道获得弥拜霉 素原料药，以降低其价格，前人尝试了很多方法，如发 酵法、全合成法 ${ }^{[5]}$, 但都未能很好地解决这一问题.

本课题组注意到依维菌素倾向于作为杀虫、杀螨 剂 $^{[6]}$, 而弥拜霉素类化合物多用于杀虫、驱虫剂 ${ }^{[7]}$. 另外

*E-mail: jinhaozhao@zju.edu.cn

Received November 10, 2011; revised January 7, 2012; published online February 27, 2012.

Project supported by the Important Technology Special Topic Project of Zhejiang Province (No. 2008C02007-3).

浙江省科技厅重大(No. 2008C02007-3)资助项目. 


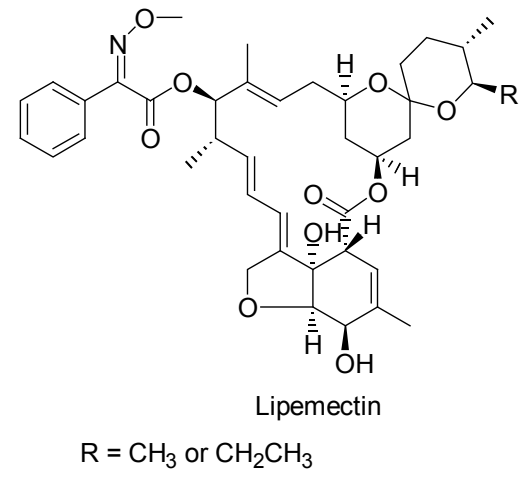

Scheme 1

依维菌素在国内多家农药公司均有售, 且价格较低. 值 得关注的是弥拜霉素与脱去两个糖基的依维菌素 (Ivermectin Aglycone)有着极其相似的结构(Scheme 2).

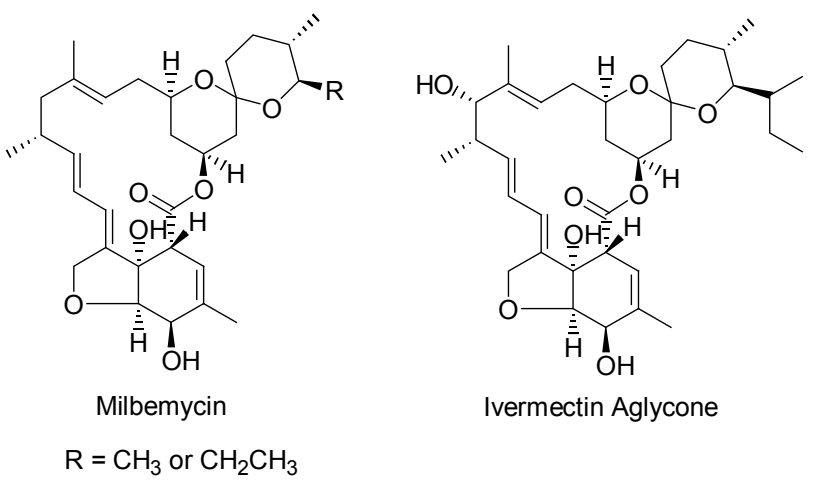

Scheme 2

为此, 本文以依维菌素为原料, 根据类同合成法和 亚结构连接法的原理, 对糖基进行水解, 脱去两个糖基, 经过合理的分子衍生设计, 合成了一系列无糖弥拜霉素 类似物 (4IIa $\sim 4 \mathrm{IId}, \mathbf{5 I I a} \sim \mathbf{5 I I d}$, Scheme 3). 另外将依维 菌素脱去一个糖基, 连接相同取代基, 合成了另一个系 列单糖弥拜需素类似物 (4Ia $\sim$ 4Id, 5Ia $\sim 5$ Id, Scheme 4). 所有目标化合物的分子结构经 ${ }^{1} \mathrm{H}$ NMR, HRMS 得到确 证, 并对其进行杀虫活性测定. 在前期工作中, 中间体 2I, 2II, 3I 和 3II 已作报道 ${ }^{[8,9]}$, 且发现 13-(2,2-二甲基)丁 酰基弥拜霉素类似物等化合物对粘虫和蚜虫具有很好 的杀虫活性. 在此次生测结果中显示化合物 5Id 5IId 几乎没有杀虫活性, 而化合物 4Ia $\sim 4$ III 表现出较好的 杀虫活性, 尤其是化合物 4III 和 4IIIb 对粘虫和蜴虫的 杀虫活性最高.

\section{1 实验部分}

\section{1 仪器和试剂}

XRC-1 显微熔点仪(北京泰克仪器有限公司), 温度 未校正; 核磁共振氢谱仪(Bruker AVANCE III 500),
TMS 为内标, $\mathrm{CDCl}_{3}$ 为溶剂; Bruker Esquire 3000 离子肼 液相色谱质谱仪. 所有试剂均为分析纯; 中间体 2I, 2II, 3I 和 3II 的制备详见参考文献 [8, 9]; 所有酰氯均按照参 考文献[10]制备.

1.2 实验步骤

1.2.1 弥拜霉素类似物 $4 \mathrm{Ia} \sim 4 \mathrm{Id}$ 和 $4 \mathrm{IIa} \sim 4 \mathrm{IId}$ 的通 用制备法

$25 \mathrm{~mL}$ 圆底烧瓶中加入 5-O-TBDMS-单糖弥拜霉素 类似物 (3I， $0.25 \mathrm{mmol}$ )或者 5-O-TBDMS-无糖弥拜霉素

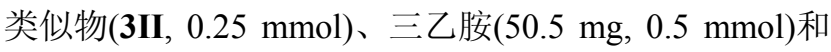
DMAP $(1.22 \mathrm{mg}, 0.01 \mathrm{mmol})$, 再加入 $8 \mathrm{~mL}$ 干燥的二氯 甲烷. 缓慢滴加 $5 \mathrm{~mL}$ 酰氯 $(0.5 \mathrm{mmol})$ 二氯甲烷溶液, 室 温下继续搅拌反应 $8 \mathrm{~h}$. 反应液用二氯甲烷 $(30 \mathrm{~mL})$ 稀释, 用稀盐酸 $(15 \mathrm{~mL} \times 3,5 \%) 、 \mathrm{NaHCO}_{3}(15 \mathrm{~mL} \times 3,5 \%)$ 溶液 和饱和食盐水 $(15 \mathrm{~mL} \times 3)$ 洗涤, 无水硫酸钠干燥、浓缩 得酯化后的产物 $\mathbf{A}$.

将上述产物 $\mathbf{A}$ 置于 $25 \mathrm{~mL}$ 圆底烧瓶中, 加入 $15 \mathrm{~mL}$ 对甲苯磺酸甲醇溶液 $\left(0.020 \mathrm{~g} \cdot \mathrm{mL}^{-1}\right)$, 室温摚拌反应 30 min. 再加入 $\mathrm{NaHCO}_{3}(30 \mathrm{~mL}, 5 \%)$ 后, 乙酸乙酯(30 $\mathrm{mL} \times 3)$ 提取, 有机层用饱和食盐水 $(20 \mathrm{~mL} \times 3)$ 洗涤, 无 水硫酸钠干燥、浓缩, 经硅胶柱 $[V$ (乙酸乙酯) $: V($ 石油 醚) $=20 ： 60$ ]纯化得目标产物 4Ia $\sim 4$ Id 和 4IIa $\sim 4$ IId.

4'-邻氯苯甲酰基弥拜霉素类似物(4Ia): 产率 $87.1 \%$. 无色晶体. m.p. $110 \sim 114{ }^{\circ} \mathrm{C} ;{ }^{1} \mathrm{H}$ NMR $\left(\mathrm{CDCl}_{3}, 500\right.$ MHz) $\delta: 7.80 \sim 7.83(\mathrm{~m}, 1 \mathrm{H}, \mathrm{H}-\mathrm{Ar}), 7.32 \sim 7.48(\mathrm{~m}, 3 \mathrm{H}$, H-Ar), $5.85 \sim 5.87$ (m, 1H, H-9), $5.73 \sim 5.77$ (m, 2H, H-10, $\mathrm{H}-11), 5.43(\mathrm{~s}, 1 \mathrm{H}, \mathrm{H}-3), 5.34 \sim 5.36(\mathrm{~m}, 1 \mathrm{H}, \mathrm{H}-19)$, $5.01 \sim 5.07$ (m, 1H, H-15), 4.93 (t, $\left.J=9.5 \mathrm{~Hz}, 1 \mathrm{H}, \mathrm{H}-4^{\prime}\right)$, $4.86 \sim 4.88(\mathrm{~m}, 1 \mathrm{H}, \mathrm{H}-1), 4.65 \sim 4.69(\mathrm{~m}, 2 \mathrm{H}, \mathrm{H}-8 \mathrm{a})$, $4.27 \sim 4.30$ (m, $1 \mathrm{H}, \mathrm{H}-5), 4.17$ (s, $1 \mathrm{H}, 7-\mathrm{OH}), 4.02 \sim 4.07$ (m, 1H, H-5'), 3.97 3.98 (m, 2H, H-6, H-13), 3.67 3.88 (m, 2H, H-17, H-3'), 3.45 (s, 3H, 3'- $-\mathrm{OCH}_{3}$ ), 3.22 3.30 (m, $2 \mathrm{H}, \mathrm{H}-2, \mathrm{H}-25), 2.53 \sim 2.56$ (m, 1H, H-12), $2.30 \sim 2.57$ (m, $5 \mathrm{H}, \mathrm{H}-16, \mathrm{H}-2$ ', H-24), 1.74 1.88 (m, 5H, 4- $\mathrm{CH}_{3}, \mathrm{H}-18$ ), $1.17 \sim 1.65\left(\mathrm{~m}, 12 \mathrm{H}, 14-\mathrm{CH}_{3}, \mathrm{H}-20, \mathrm{H}-26, \mathrm{H}-27, \mathrm{H}-22\right.$, $\mathrm{H}-23), 1.16 \sim 1.26\left(\mathrm{~m}, 6 \mathrm{H}, 5^{\prime}-\mathrm{CH}_{3}, 12-\mathrm{CH}_{3}\right), 0.79 \sim 0.96$ (m, 9H, 24,26,28- $\mathrm{CH}_{3}$ ). HRMS (ESI) calcd for $\mathrm{C}_{48} \mathrm{H}_{65} \mathrm{Cl}-$ $\mathrm{O}_{12} \mathrm{Na}(\mathrm{M}+\mathrm{Na})^{+}$891.4062, found 891.4058.

4'-邻甲氧苯甲酰基弥拜霉素类似物 (4Ib): 产率 $75.3 \%$. 无色晶体, m.p. $103 \sim 106{ }^{\circ} \mathrm{C} ;{ }^{1} \mathrm{H} \mathrm{NMR}\left(\mathrm{CDCl}_{3}\right.$, $500 \mathrm{MHz}) \delta: 7.78$ (d, $J=8.0 \mathrm{~Hz}, 1 \mathrm{H}, \mathrm{H}-6-\mathrm{Ar}), 7.47$ (d, $J=$ $8.6 \mathrm{~Hz}, 2 \mathrm{H}, \mathrm{H}-4,5-\mathrm{Ar}), 6.98$ (d, $J=7.9 \mathrm{~Hz}, 1 \mathrm{H}, \mathrm{H}-3-\mathrm{Ar}$ ), $5.86 \sim 5.87$ (m, 1H, H-9), 5.71 5.81 (m, 2H, H-10, H-11), 5.43 (s, 1H, H-3), $5.32 \sim 5.34$ (m, 1H, H-19), $5.00 \sim 5.02$ (m, 1H, H-15), 4.92 (t, $J=9.5 \mathrm{~Hz}, 1 \mathrm{H}, \mathrm{H}-4$ '), $4.85 \sim 4.88$ 


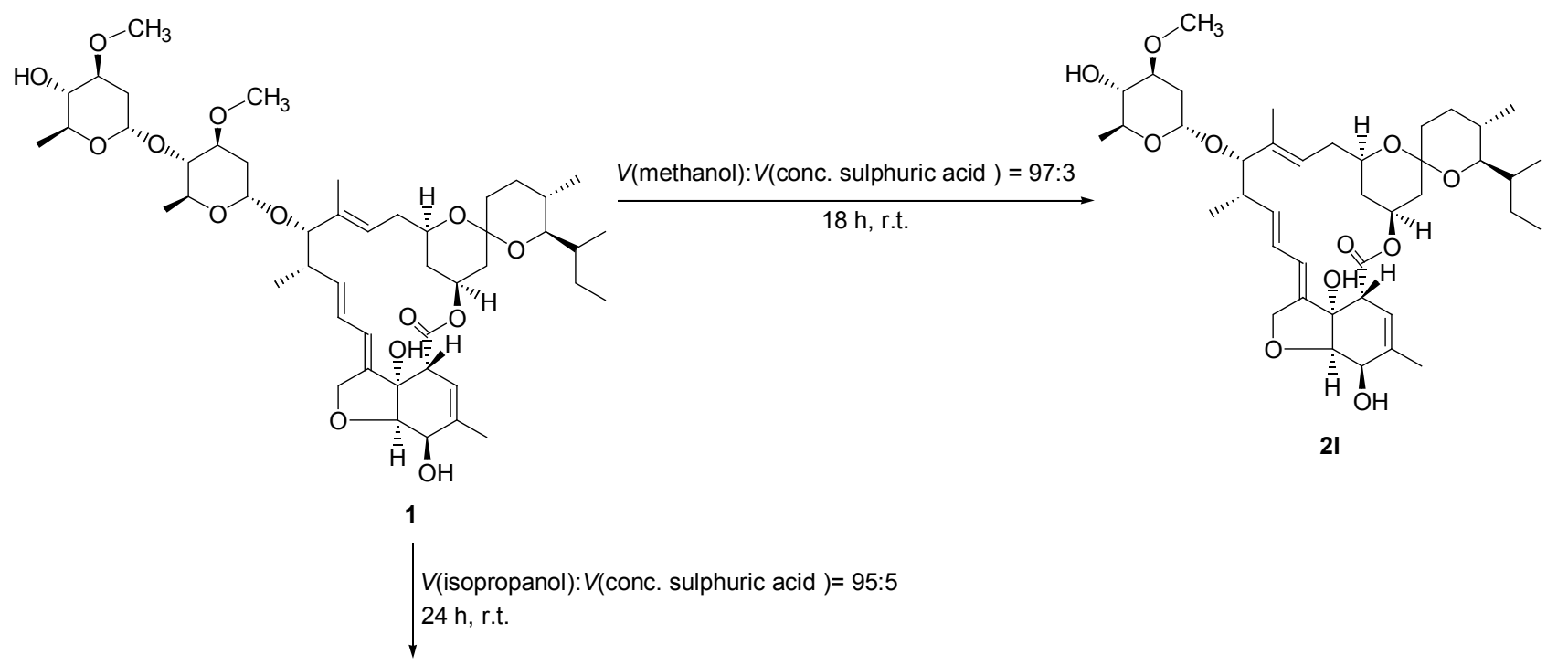

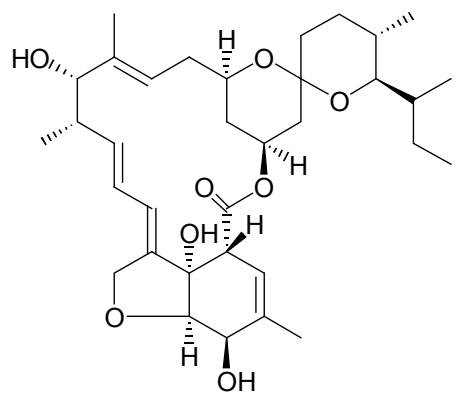

2II

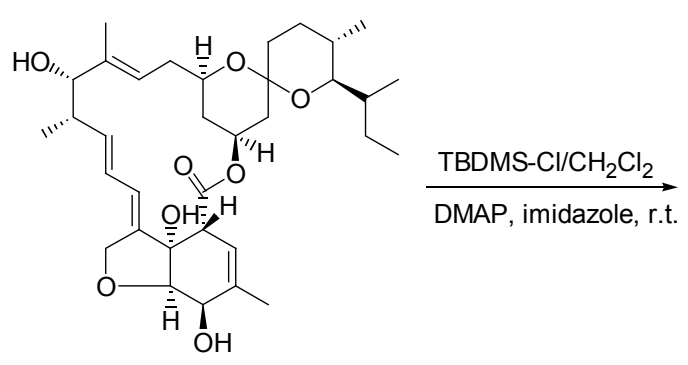

2II

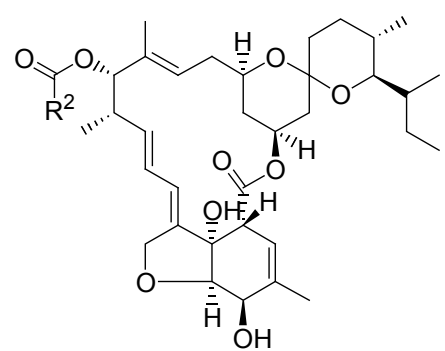

4lla $\sim$ 4lld
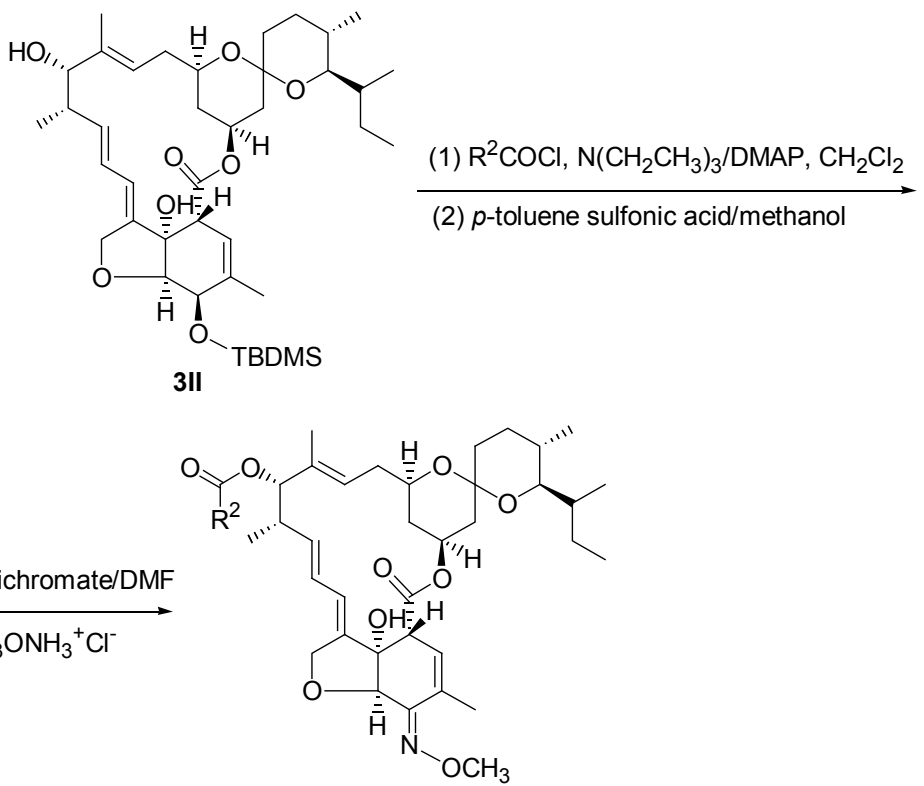

$5 \mathrm{lla} \sim 5 \mathrm{lld}$

Scheme 3

(m, 1H, H-1'), $4.63 \sim 4.64$ (m, 2H, H-8a), 4.26 4.29 (m, 1H, H-5), 4.11 (s, 1H, 7-OH), 4.07 4.08 (m, 1H, H-5'), $3.96 \sim 3.98$ (m, 2H, H-6, H-13), 3.90 (s, 3H, Ar-2- $-\mathrm{OCH}_{3}$ ), $3.69 \sim 3.83\left(\mathrm{~m}, 2 \mathrm{H}, \mathrm{H}-17, \mathrm{H}-3^{\prime}\right), 3.45$ (s, 3H, 3'- $\left.-\mathrm{OCH}_{3}\right)$, $3.22 \sim 3.30(\mathrm{~m}, 2 \mathrm{H}, \mathrm{H}-2, \mathrm{H}-25), 2.53 \sim 2.56$ (m, 1H, H-12),
$2.17 \sim 2.35$ (m, 5H, H-16, H-2', H-24), 1.73 1.87 (m, 5H, 4- $\left.\mathrm{CH}_{3}, \mathrm{H}-18\right), 1.34 \sim 1.68\left(\mathrm{~m}, 12 \mathrm{H}, 14-\mathrm{CH}_{3}, \mathrm{H}-20, \mathrm{H}-26\right.$, $\mathrm{H}-27, \mathrm{H}-22, \mathrm{H}-23), 0.93 \sim 0.99$ (m, 6H, 5'- $\mathrm{CH}_{3}, 2-\mathrm{CH}_{3}$ ), $0.73 \sim 0.80\left(\mathrm{~m}, 9 \mathrm{H}, 24,26,28-\mathrm{CH}_{3}\right)$. HRMS (ESI) calcd for $\mathrm{C}_{49} \mathrm{H}_{68} \mathrm{O}_{13} \mathrm{Na}(\mathrm{M}+\mathrm{Na})^{+}$887.4558, found 887.4554. 

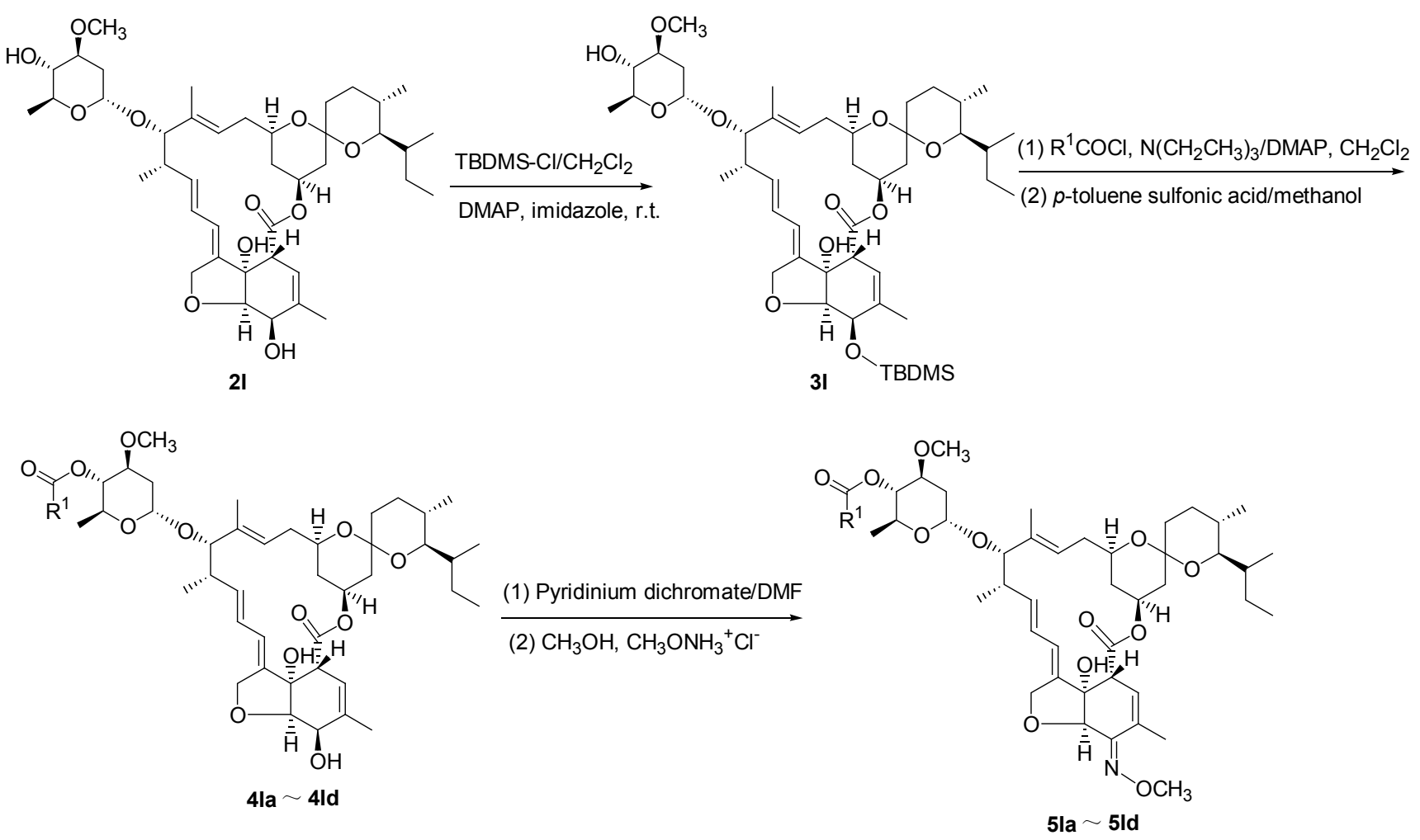

Scheme 4

4'-邻甲苯甲酰基弥拜霉素类似物 (4Ic): 产率 $80.9 \%$. 无色晶体, m.p. $145 \sim 147{ }^{\circ} \mathrm{C} ;{ }^{1} \mathrm{H}$ NMR $\left(\mathrm{CDCl}_{3}\right.$, $500 \mathrm{MHz}) \delta: 7.91 \sim 7.81(\mathrm{~m}, 1 \mathrm{H}, \mathrm{H}-6-\mathrm{Ar}), 7.26 \sim 7.35(\mathrm{~m}$, 1H, H-3,4-Ar), $7.02 \sim 7.04$ (m, 1H, H-5-Ar), 5.88 5.90 (m, 1H, H-9), 5.74 5.78 (m, 2H, H-10, H-11), 5.43 (s, $1 \mathrm{H}, \mathrm{H}-3), 5.37 \sim 5.42(\mathrm{~m}, 1 \mathrm{H}, \mathrm{H}-19), 5.01 \sim 5.09(\mathrm{~m}, 1 \mathrm{H}$, H-15), $4.90 \sim 4.96$ (t, $\left.J=9.5 \mathrm{~Hz}, 1 \mathrm{H}, \mathrm{H}-4^{\prime}\right), 4.88 \sim 4.90(\mathrm{~m}$, $\left.1 \mathrm{H}, \mathrm{H}-1^{\prime}\right), 4.68 \sim 4.69$ (m, 2H, H-8a), $4.22 \sim 4.30(\mathrm{~m}, 1 \mathrm{H}$, $\mathrm{H}-5), 4.15(\mathrm{~s}, 1 \mathrm{H}, 7-\mathrm{OH}), 4.07 \sim 4.10\left(\mathrm{~m}, 1 \mathrm{H}, \mathrm{H}-5^{\prime}\right)$, $3.97 \sim 3.99$ (m, 2H, H-6, H-13), 3.82 3.86 (m, 1H, H-17), $3.67 \sim 3.69\left(\mathrm{~m}, 1 \mathrm{H}, \mathrm{H}-3^{\prime}\right), 3.43\left(\mathrm{~s}, 3 \mathrm{H}, 3^{\prime}-\mathrm{OCH}_{3}\right), 3.22 \sim$ 3.30 (m, 2H, H-2, H-25), 2.56 2.59 (m, 1H, H-12), 2.62 (s, 3H, Ar-2- $\mathrm{CH}_{3}$ ), $2.30 \sim 2.55$ (m, 5H, H-16, H-2', H-24), $1.77 \sim 1.99\left(\mathrm{~m}, 5 \mathrm{H}, 4-\mathrm{CH}_{3}, \mathrm{H}-18\right), 1.34 \sim 1.65(\mathrm{~m}, 12 \mathrm{H}$, 14- $\mathrm{CH}_{3}, \mathrm{H}-20, \mathrm{H}-26, \mathrm{H}-27, \mathrm{H}-22, \mathrm{H}-23$ ), $1.17 \sim 1.23$ (m, $6 \mathrm{H}, 5$ ' $\left.-\mathrm{CH}_{3}, 12-\mathrm{CH}_{3}\right), 0.79 \sim 0.96\left(\mathrm{~m}, 9 \mathrm{H}, 24,26,28-\mathrm{CH}_{3}\right)$. HRMS (ESI) calcd for $\mathrm{C}_{49} \mathrm{H}_{68} \mathrm{O}_{12} \mathrm{Na}(\mathrm{M}+\mathrm{Na})^{+}$871.4609, found 871.4611 .

4'-2-对氯苯基-3-甲基丁酰基弥拜霉素类似物(4Id): 产率 59.4\%. 无色晶体, m.p. $170 \sim 173{ }^{\circ} \mathrm{C}$; ${ }^{1} \mathrm{H}$ NMR $\left(\mathrm{CDCl}_{3}, 500 \mathrm{MHz}\right) \delta: 7.72 \sim 7.31(\mathrm{~m}, 4 \mathrm{H}, \mathrm{Ar}-\mathrm{H}), 5.82(\mathrm{~m}$, $1 \mathrm{H}, \mathrm{H}-9), 5.72 \sim 5.74(\mathrm{~m}, 2 \mathrm{H}, \mathrm{H}-10, \mathrm{H}-11), 5.33 \sim 5.34$ (m, 2H, H-3, H-19), 4.96 4.98 (m, 1H, H-15), 4.81 4.86 (m, 1H, H-1'), $4.57 \sim 4.70$ (m, 3H, H-8a, H-4'), 4.44 4.48 (m,
$1 \mathrm{H}, \mathrm{H}-5), 4.25$ (s, 1H, 7-OH), 3.92 $3.93(\mathrm{~m}, 2 \mathrm{H}, \mathrm{H}-5$ ', $\mathrm{H}-3), 4.83 \sim 4.85(\mathrm{~m}, 1 \mathrm{H}, \mathrm{H}-6), 3.63 \sim 3.70(\mathrm{~m}, 3 \mathrm{H}, \mathrm{CH}-$ $\left.\mathrm{C}=\mathrm{O}, \mathrm{H}-17, \mathrm{H}-3^{\prime}\right), 3.38 \sim 3.39$ (m, 1H, H-2), $3.29(\mathrm{~s}, 3 \mathrm{H}$, $\left.3^{\prime}-\mathrm{OCH}_{3}\right), 3.20 \sim 3.24(\mathrm{~m}, 1 \mathrm{H}, \mathrm{H}-25), 2.52 \sim 2.54(\mathrm{~m}, 1 \mathrm{H}$, H-12), $2.22 \sim 2.32$ (m, 5H, H-16, H-2', H-24), $1.80 \sim 2.00$ (m, 5H, 4- $\left.\mathrm{CH}_{3}, \mathrm{H}-18\right), 1.33 \sim 1.66$ (m, $14 \mathrm{H}, 14-\mathrm{CH}_{3}, \mathrm{H}-20$, $\mathrm{H}-26, \mathrm{H}-27, \mathrm{H}-22, \mathrm{H}-23), 1.03 \sim 1.25\left(\mathrm{~m}, 12 \mathrm{H},\left(\mathrm{CH}_{3}\right)_{2} \mathrm{CH}-\right.$ $\left.\mathrm{C}=\mathrm{O}, \quad 5 '-\mathrm{CH}_{3}, \quad 12-\mathrm{CH}_{3}\right), 0.77 \sim 0.95(\mathrm{~m}, 9 \mathrm{H}, 24,26,28-$ $\mathrm{CH}_{3}$ ). HRMS (ESI) calcd for $\mathrm{C}_{52} \mathrm{H}_{73} \mathrm{ClO}_{12} \mathrm{Na}(\mathrm{M}+\mathrm{Na})^{+}$ 947.4688, found 947.4684.

13-邻氯苯甲酰基弥拜霉素类似物 (4IIa): 产率 $81.6 \%$. 白色固体, m.p. $110 \sim 114{ }^{\circ} \mathrm{C} ;{ }^{1} \mathrm{H}$ NMR $\left(\mathrm{CDCl}_{3}\right.$, $500 \mathrm{MHz}) \delta: 7.90 \sim 7.93(\mathrm{~m}, 1 \mathrm{H}, \mathrm{H}-6-\mathrm{Ar}), 7.44 \sim 7.45(\mathrm{~m}$, $2 \mathrm{H}, \mathrm{H}-3,4-\mathrm{Ar}$ ), $7.37 \sim 7.40$ (m, 1H, H-5-Ar), 5.85 5.88 (m, 1H, H-9), 5.79 5.83 (m, 2H, H-10, H-11), 5.42 (s, $2 \mathrm{H}, \mathrm{H}-3, \mathrm{H}-13$ ), $5.27 \sim 5.30$ (m, 1H, H-19), $5.15 \sim 5.19$ (m, $1 \mathrm{H}, \mathrm{H}-15), 4.65 \sim 4.69(\mathrm{~m}, 2 \mathrm{H}, \mathrm{H}-8 \mathrm{a}), 4.28 \sim 4.31(\mathrm{~m}, 1 \mathrm{H}$, H-5), 4.08 (s, 1H, 7-OH), 3.96 3.97 (d, $J=6.5 \mathrm{~Hz}, 1 \mathrm{H}$, H-6), $3.59 \sim 3.62$ (m, 1H, H-17), $3.27 \sim 3.31$ (dd, $J=2.0$, $4.5 \mathrm{~Hz}, 1 \mathrm{H}, \mathrm{H}-2), 3.15$ (d, $J=8.0 \mathrm{~Hz}, 1 \mathrm{H}, \mathrm{H}-25), 2.74 \sim$ 2.78 (m, 1H, H-12), 2.49 (d, $J=8.5 \mathrm{~Hz}, 1 \mathrm{H}, \mathrm{H}-24), 2.26 \sim$ 2.29 (m, 2H, H-16), 1.74 2.04 (m, 5H, 4- $\mathrm{CH}_{3}, \mathrm{H}-18$ ), $1.24 \sim 1.86\left(\mathrm{~m}, 12 \mathrm{H}, 14-\mathrm{CH}_{3}, \mathrm{H}-20, \mathrm{H}-26, \mathrm{H}-27, \mathrm{H}-22\right.$, $\mathrm{H}-23), 1.16 \sim 1.18\left(\mathrm{~m}, 3 \mathrm{H}, 12-\mathrm{CH}_{3}\right), 0.76 \sim 0.87(\mathrm{~m}, 9 \mathrm{H}$, 
24,26,28- $\left.\mathrm{CH}_{3}\right) ;{ }^{13} \mathrm{C} \mathrm{NMR}\left(100 \mathrm{MHz}, \mathrm{CDCl}_{3}\right) \delta: 168.59$ $(\mathrm{C}-1), 165.31,166.10(\mathrm{OC}=\mathrm{O}, \mathrm{ArC}=\mathrm{O}), 139.80,135.24$, $133.00,132.76,132.01,131.63,130.01,129.85,129.74$, 129.39 (C-1,2,3,4,6-Ar, C-8, C-11, C-14, C-4), 125.80, 121.73 (C-5-Ar, C-10), 115.21 (C-9), 113.08, 112.92 (C-3 or C-15), 99.14 (C-21), 75.29, 74.15, 74.10, 71.88 (C-25, C-13, C-7, C-6), 63.65, 63.39, 62.68, 62.13 (C-8a, C-19, C-17, C-5), 40.65, 36.25, 33.87, 31.72 (C-2, C-12, C-20, C-18), 27.56, 27.49 (C-26, C-16, C-22), 29.11, 26.75, 26.23 (C-24), 24.67, 23.94 (C-23), 17.60, 15.22, 14.90, 12.95, 12.42, 10.31, 9.57 (C-27, C-4a, C-12a, C-24a, $\mathrm{C}-14 \mathrm{a}, \mathrm{C}-26 \mathrm{a}, \mathrm{C}-28$ ). HRMS (ESI) calcd for $\mathrm{C}_{41} \mathrm{H}_{53} \mathrm{Cl}-$ $\mathrm{O}_{9} \mathrm{Na}(\mathrm{M}+\mathrm{Na})^{+}$747.3276, found 747.3280.

13-邻甲氧基甲苯甲酰基弥拜霉素类似物(4IIb): 产 率 79.4\%. 无色油状物. ${ }^{1} \mathrm{H} \mathrm{NMR}\left(\mathrm{CDCl}_{3}, 500 \mathrm{MHz}\right) \delta$ : $8.04 \sim 8.06$ (m, 1H, H-6-Ar), $7.73 \sim 7.75$ (m, 1H, H-4-Ar), $7.20 \sim 7.25$ (m, 2H, H-3,5-Ar), 5.86 5.89 (m, 1H, H-9), $5.80 \sim 5.85$ (m, 2H, H-10, H-11), 5.61 (s, 1H, H-13), 5.38 $(\mathrm{s}, 1 \mathrm{H}, \mathrm{H}-3), 5.30 \sim 5.32(\mathrm{~m}, 1 \mathrm{H}, \mathrm{H}-19), 5.16 \sim 5.19(\mathrm{~m}$, $1 \mathrm{H}, \mathrm{H}-15), 4.58 \sim 4.60(\mathrm{~m}, 2 \mathrm{H}, \mathrm{H}-8 \mathrm{a}), 4.29 \sim 4.33(\mathrm{~m}, 1 \mathrm{H}$, H-5), 4.22 (d, J=6.5 Hz, 1H, H-6), 4.08 (s, 1H, 7-OH), 3.89 (s, 3H, Ar-2- $\left.\mathrm{OCH}_{3}\right), 3.60 \sim 3.62(\mathrm{~m}, 1 \mathrm{H}, \mathrm{H}-17), 3.47$ (dd, $J=2.0,4.5 \mathrm{~Hz}, 1 \mathrm{H}, \mathrm{H}-2), 3.13$ (d, $J=8.0 \mathrm{~Hz}, 1 \mathrm{H}$, $\mathrm{H}-25), 2.71 \sim 2.73(\mathrm{~m}, 1 \mathrm{H}, \mathrm{H}-12), 2.52$ (d, $J=8.5 \mathrm{~Hz}, 1 \mathrm{H}$, $\mathrm{H}-24), 2.26 \sim 2.29(\mathrm{~m}, 2 \mathrm{H}, \mathrm{H}-16), 1.78 \sim 2.04(\mathrm{~m}, 5 \mathrm{H}$, 4- $\left.\mathrm{CH}_{3}, \mathrm{H}-18\right), 1.26 \sim 1.66$ (m, 12H, 14- $\mathrm{CH}_{3}, \mathrm{H}-20, \mathrm{H}-26$, $\mathrm{H}-27, \mathrm{H}-22, \mathrm{H}-23), 1.13 \sim 1.16\left(\mathrm{~m}, 3 \mathrm{H}, 12-\mathrm{CH}_{3}\right), 0.75 \sim$ 0.87 (m, 9H, 24,26,28- $\left.\mathrm{CH}_{3}\right) ;{ }^{13} \mathrm{C} \mathrm{NMR}\left(100 \mathrm{MHz}, \mathrm{CDCl}_{3}\right)$ $\delta: 168.61(\mathrm{C}-1), 165.30,166.23(\mathrm{OC}=\mathrm{O}, \mathrm{ArC}=\mathrm{O})$, 158.91 (C-2-Ar), 135.24, 134.63, 132.76, 130.01, 129.85, 129.39 (C-4,6-Ar, C-8, C-11, C-14, C-4), 121.73, 120.9, 120.6 (C-1,5-Ar, C-10), 115.21, 114.23 (C-3-Ar, C-9), 113.08, 112.92 (C-3 or C-15), 99.14 (C-21), 75.29, 74.15, 74.10, 71.88 (C-25, C-13, C-7, C-6), 63.65, 63.39, 62.68, 62.13 (C-8a, C-19, C-17, C-5), $55.90\left(\mathrm{OCH}_{3}\right), 40.65$, 36.25, 33.87, 31.72 (C-2, C-12, C-20, C-18), 27.56, 27.49 (C-26, C-16, C-22), 29.11, 26.75, 26.23 (C-24), 24.67, 23.94 (C-23), 17.60, 15.22, 14.90, 12.95, 12.42, 10.31, 9.57 (C-27, C-4a, C-12a, C-24a, C-14a, C-26a, C-28). HRMS (ESI) calcd for $\mathrm{C}_{42} \mathrm{H}_{56} \mathrm{O}_{10} \mathrm{Na}(\mathrm{M}+\mathrm{Na})^{+} 743.3771$, found 743.3775 .

13-邻甲苯甲酰基弥拜霉素类似物 (4IIc): 产率 $80.6 \%$. 无色晶体, m.p. $100 \sim 102{ }^{\circ} \mathrm{C} ;{ }^{1} \mathrm{H}$ NMR $\left(\mathrm{CDCl}_{3}\right.$, $500 \mathrm{MHz}) \delta: 8.03 \sim 8.05(\mathrm{~m}, 1 \mathrm{H}, \mathrm{H}-6-\mathrm{Ar}), 7.44 \sim 7.46(\mathrm{~m}$, $1 \mathrm{H}, \mathrm{H}-4-\mathrm{Ar}), 7.28 \sim 7.32(\mathrm{~m}, 2 \mathrm{H}, \mathrm{H}-3,5-\mathrm{Ar}), 5.89 \sim 5.91$ (m, 1H, H-9), 5.77 5.87 (m, 2H, H-10, H-11), 5.43 (s, $1 \mathrm{H}, \mathrm{H}-3), 5.39$ (s, 1H, H-13), 5.30 5.32 (m, 1H, H-19), $5.14 \sim 5.16(\mathrm{~m}, 1 \mathrm{H}, \mathrm{H}-15), 4.66 \sim 4.68(\mathrm{~m}, 2 \mathrm{H}, \mathrm{H}-8 \mathrm{a})$, $4.30 \sim 4.33$ (m, 1H, H-5), 4.08 (s, 1H, 7-OH), 3.97 (d, J= $6.5 \mathrm{~Hz}, 1 \mathrm{H}, \mathrm{H}-6), 3.56 \sim 3.58$ (m, 1H, H-17), 3.29 (dd, $J=$ $2.0,4.5 \mathrm{~Hz}, 1 \mathrm{H}, \mathrm{H}-2), 3.13$ (d, $J=8.0 \mathrm{~Hz}, 1 \mathrm{H}, \mathrm{H}-25)$, $2.75 \sim 2.78(\mathrm{~m}, 1 \mathrm{H}, \mathrm{H}-12), 2.64\left(\mathrm{~s}, 3 \mathrm{H}, \mathrm{Ar}-2-\mathrm{CH}_{3}\right), 2.50(\mathrm{~d}$, $J=8.5 \mathrm{~Hz}, 1 \mathrm{H}, \mathrm{H}-24), 2.25 \sim 2.27$ (m, 2H, H-16), $1.75 \sim$ $2.04\left(\mathrm{~m}, 5 \mathrm{H}, 4-\mathrm{CH}_{3}, \mathrm{H}-18\right), 1.26 \sim 1.64\left(\mathrm{~m}, 12 \mathrm{H}, 14-\mathrm{CH}_{3}\right.$, $\mathrm{H}-20, \mathrm{H}-26, \mathrm{H}-27, \mathrm{H}-22, \mathrm{H}-23), 1.13 \sim 1.15(\mathrm{~m}, 3 \mathrm{H}$, $\left.12-\mathrm{CH}_{3}\right), 0.74 \sim 0.88\left(\mathrm{~m}, 9 \mathrm{H}, 24,26,28-\mathrm{CH}_{3}\right)$. HRMS (ESI) calcd for $\mathrm{C}_{42} \mathrm{H}_{56} \mathrm{O}_{9} \mathrm{Na}(\mathrm{M}+\mathrm{Na})^{+} 727.3822$, found 727.3819 .

13-(2-对氯苯基)-3-甲基丁酰基弥拜霉素类似物 (4IId): 无色晶体, 产率 68.6\%. m.p. $122 \sim 125{ }^{\circ} \mathrm{C} ;{ }^{1} \mathrm{H}$ NMR $\left(\mathrm{CDCl}_{3}, 500 \mathrm{MHz}\right) \delta: 7.72 \sim 7.31(\mathrm{~m}, 4 \mathrm{H}, \mathrm{ArH})$, $5.85 \sim 5.88$ (m, 1H, H-9), 5.64 5.79 (m, 2H, H-10, H-11), 5.41 (s, 1H, H-3), 5.26 (m, 1H, H-19), 5.18 (s, 1H, H-13), 5.03 (s, 1H, H-15), $4.62 \sim 4.28$ (m, 2H, H-8a), 4.28 4.31 (m, 1H, H-5), 3.93 (d, J=6.5 Hz, 1H, H-6), 3.66 3.69 $(\mathrm{m}, 2 \mathrm{H}, \mathrm{H}-17, \mathrm{CHC}=\mathrm{O}), 3.27(\mathrm{dd}, J=2.0,4.5 \mathrm{~Hz}, 1 \mathrm{H}$, $\mathrm{H}-2), 3.17$ (d, $J=8.0 \mathrm{~Hz}, 1 \mathrm{H}, \mathrm{H}-25), 2.52 \sim 2.56(\mathrm{~m}, 1 \mathrm{H}$, H-12), $2.22 \sim 2.32$ (m, 5H, H16, H-2', H-24), $1.80 \sim 2.00$ (m, 5H, 4- $\left.\mathrm{CH}_{3}, \mathrm{H}-18\right), 1.33 \sim 1.66$ (m, 14H, 14- $\mathrm{CH}_{3}, \mathrm{H}-20$, $\mathrm{H}-26, \mathrm{H}-27, \mathrm{H}-22, \mathrm{H}-23), 1.03 \sim 1.25\left(\mathrm{~m}, 12 \mathrm{H},\left(\mathrm{CH}_{3}\right)_{2} \mathrm{CH}-\right.$ $\left.\mathrm{C}=\mathrm{O}, \quad 5 '-\mathrm{CH}_{3}, \quad 12-\mathrm{CH}_{3}\right), 0.77 \sim 0.95(\mathrm{~m}, 9 \mathrm{H}, 24,26$, 28- $\mathrm{CH}_{3}$ ). HRMS (ESI) calcd for $\mathrm{C}_{45} \mathrm{H}_{61} \mathrm{ClO}_{9} \mathrm{Na}(\mathrm{M}+\mathrm{Na})^{+}$ 803.3902, found 803.3906.

\subsection{2 弥拜霉素类似物 $5 \mathrm{Ia} \sim 5 \mathrm{Id}$ 和 $\mathbf{5 I I a} \sim 5 \mathrm{IId}$ 的通 用制备法}

$50 \mathrm{~mL}$ 圆底烧瓶中加入酯化的单糖或无糖弥拜霉素 类似物 $(0.25 \mathrm{mmol})$ 和重铬酸吡定盐 $(188 \mathrm{mg}, 0.5 \mathrm{mmol})$, 加入 $30 \mathrm{~mL}$ 干燥 $\mathrm{DMF}$ 作为溶剂, 室温摚拌反应 $40 \mathrm{~min}$. 反应液中加入 $100 \mathrm{~mL}$ 水，再用乙醚 $(3 \times 50 \mathrm{~mL})$ 提取，有 机层用稀盐酸 $(20 \mathrm{~mL} \times 3,5 \%)$ 洗涤, 无水硫酸钠干燥、 浓缩得氧化产物 $\mathbf{B}$.

$25 \mathrm{~mL}$ 圆底烧瓶中加入产物 $\mathbf{B}(0.25 \mathrm{mmol})$ 和甲氧胺 基盐酸盐(42 mg, $0.5 \mathrm{mmol}$ ), 加入 $20 \mathrm{~mL}$ 甲醇作为溶剂, 室温搅拌反应 $1 \mathrm{~h}$. 反应液中加入 $30 \mathrm{~mL}$ 水, 再用乙酸乙 酯(30 mL $\times 3)$ 提取，有机层用稀盐酸 $(20 \mathrm{~mL} \times 3,5 \%)$ 洗 涤, 无水硫酸钠干燥、浓缩, 经硅胶柱 $[V($ 乙酸乙酯 $)$ : $V($ 石油醚 $)=20 ： 100)$ 纯化得目标产物 5 Ia $\sim 5$ Id 和 5IIa $\sim 5$ IId.

5-甲氧亚氨基-4'-邻氯苯甲酰基弥拜需素类似物 (5Ia): 产率 77.1\%. 无色晶体, m.p. 89 92 ${ }^{\circ} \mathrm{C} ;{ }^{1} \mathrm{H}$ NMR 
$\left(\mathrm{CDCl}_{3}, 500 \mathrm{MHz}\right) \delta: 7.80 \sim 7.83(\mathrm{~m}, 1 \mathrm{H}, \mathrm{H}-6-\mathrm{Ar}), 7.41 \sim$ 7.43 (m, 2H, H-3,4-Ar), $7.29 \sim 7.33$ (m, 1H, H-5-Ar), 5.92 (d, $J=15.0 \mathrm{~Hz}, 1 \mathrm{H}, \mathrm{H}-9), 5.75 \sim 5.79$ (m, 3H, H-3, H-10, $\mathrm{H}-11), 5.37 \sim 5.40(\mathrm{~m}, 1 \mathrm{H}, \mathrm{H}-19), 5.00 \sim 5.03(\mathrm{~m}, 1 \mathrm{H}$, H-15), 4.93 (t, $J=9.5 \mathrm{~Hz}, 1 \mathrm{H}, \mathrm{H}-4), 4.88 \sim 4.91(\mathrm{~m}, 1 \mathrm{H}$, H-1'), $4.60 \sim 4.63(\mathrm{~m}, 2 \mathrm{H}, \mathrm{H}-8 \mathrm{a}), 4.56(\mathrm{~s}, 1 \mathrm{H}, 7-\mathrm{OH})$, $4.07 \sim 4.09(\mathrm{~m}, 1 \mathrm{H}, \mathrm{H}-5), 4.00\left(\mathrm{~s}, 3 \mathrm{H}, \mathrm{NOCH}_{3}\right), 3.97 \sim$ 3.99 (m, 2H, H-6, H-13), 3.67 3.84 (m, 2H, H-17, H-3'), $3.45\left(\mathrm{~s}, 3 \mathrm{H}, 3 \mathrm{\prime}-\mathrm{OCH}_{3}\right), 3.38$ (t, $\left.J=2.5 \mathrm{~Hz}, 1 \mathrm{H}, \mathrm{H}-2\right), 3.25 \sim$ 3.27 (m, 1H, H-25), $2.54 \sim 2.58(\mathrm{~m}, 1 \mathrm{H}, \mathrm{H}-12), 2.01 \sim 2.38$ (m, 5H, H-16, H-2', H-24), $1.76 \sim 1.90\left(\mathrm{~m}, 5 \mathrm{H}, 4-\mathrm{CH}_{3}\right.$, $\mathrm{H}-18), 1.40 \sim 1.69\left(\mathrm{~m}, 12 \mathrm{H}, 14-\mathrm{CH}_{3}, \mathrm{H}-20, \mathrm{H}-26, \mathrm{H}-27\right.$, $\mathrm{H}-22, \mathrm{H}-23), 1.18 \sim 1.22\left(\mathrm{~m}, 6 \mathrm{H}, 5\right.$ '- $\left.\mathrm{CH}_{3}, 12-\mathrm{CH}_{3}\right), 0.79 \sim$ $0.96\left(\mathrm{~m}, 9 \mathrm{H}, 24,25,28-\mathrm{CH}_{3}\right)$. HRMS (ESI) calcd for $\mathrm{C}_{49} \mathrm{H}_{66} \mathrm{ClNO}_{12} \mathrm{Na}(\mathrm{M}+\mathrm{Na})^{+}$918.4171, found 918.4175.

5-甲氧亚氨基-4'-对甲苯甲酰基弥拜霉素类似物 (5Ib): 产率 68.0\%. 无色晶体, m.p. 97 99 ${ }^{\circ} \mathrm{C} ;{ }^{1} \mathrm{H}$ NMR $\left(\mathrm{CDCl}_{3}, 500 \mathrm{MHz}\right) \delta: 7.98$ (d, $\left.J=7.6 \mathrm{~Hz}, 2 \mathrm{H}, \mathrm{H}-2,6-\mathrm{Ar}\right)$, 7.26 (d, $J=7.9 \mathrm{~Hz}, 2 \mathrm{H}, \mathrm{H}-3,5-\mathrm{Ar}), 5.94$ (d, $J=14.9 \mathrm{~Hz}$, $1 \mathrm{H}, \mathrm{H}-9), 5.76 \sim 5.81(\mathrm{~m}, 3 \mathrm{H}, \mathrm{H}-3, \mathrm{H}-10, \mathrm{H}-11), 5.41 \sim$ 5.43 (m, 1H, H-19), 5.02 (d, 1H, H-15), 4.92 (t, $J=9.5 \mathrm{~Hz}$, $\left.1 \mathrm{H}, \mathrm{H}-4{ }^{\prime}\right), 4.87 \sim 4.89$ (m, 1H, H-1'), $4.67 \sim 4.58(\mathrm{~m}, 2 \mathrm{H}$, H-8a), 4.58 (s, 1H, 7-OH), $4.01 \sim 4.03$ (m, 1H, H-5'), $3.99 \sim 4.00(\mathrm{~m}, 2 \mathrm{H}, \mathrm{H}-6, \mathrm{H}-13), 3.99\left(\mathrm{~s}, 3 \mathrm{H}, \mathrm{NOCH}_{3}\right)$, $3.69 \sim 3.84$ (m, 2H, H-17, H-3'), 3.41 (s, 3H, 3'- $\mathrm{OCH}_{3}$ ), 3.39 (t, $J=2.5 \mathrm{~Hz}, 1 \mathrm{H}, \mathrm{H}-2), 3.22 \sim 3.25$ (m, 1H, H-25), $2.43\left(\mathrm{~s}, 3 \mathrm{H}, \mathrm{Ar}-\mathrm{CH}_{3}\right), 2.56 \sim 2.59(\mathrm{~m}, 1 \mathrm{H}, \mathrm{H}-12), 2.28 \sim$ $2.38(\mathrm{~m}, 5 \mathrm{H}, \mathrm{H}-16, \mathrm{H}-2 \mathrm{\prime}, \mathrm{H}-24), 1.74 \sim 2.00(\mathrm{~m}, 5 \mathrm{H}$, $\left.4-\mathrm{CH}_{3}, \mathrm{H}-18\right), 1.39 \sim 1.68\left(\mathrm{~m}, 12 \mathrm{H}, 14-\mathrm{CH}_{3}, \mathrm{H}-20, \mathrm{H}-26\right.$, $\mathrm{H}-27, \mathrm{H}-22, \mathrm{H}-23), 1.18 \sim 1.22\left(\mathrm{~m}, 6 \mathrm{H}, 5{ }^{\prime}-\mathrm{CH}_{3}, 12-\mathrm{CH}_{3}\right)$, $0.79 \sim 0.96\left(\mathrm{~m}, 9 \mathrm{H}, 24,26,28-\mathrm{CH}_{3}\right)$. HRMS (ESI) calcd for $\mathrm{C}_{50} \mathrm{H}_{69} \mathrm{NO}_{12} \mathrm{Na}(\mathrm{M}+\mathrm{Na})^{+}$898.4718, found 898.4723.

5-甲氧亚氨基-4'-邻甲苯甲酰基弥拜霉素类似物 (5Ic): 产率 77.8\%. 无色晶体, m.p. 112 $1165{ }^{\circ} \mathrm{C} ;{ }^{1} \mathrm{H}$ $\mathrm{NMR}\left(\mathrm{CDCl}_{3}, 500 \mathrm{MHz}\right) \delta: 7.90(\mathrm{~d}, J=8.5 \mathrm{~Hz}, 1 \mathrm{H}$, H-6-Ar), 7.40 (d, $J=8.0 \mathrm{~Hz}, 1 \mathrm{H}, \mathrm{H}-4-\mathrm{Ar}), 7.26 \sim 7.29$ (m, 2H, H-3,5-Ar), 5.93 (d, $J=15.0 \mathrm{~Hz}, 1 \mathrm{H}, \mathrm{H}-9), 5.75 \sim 5.79$ (m, 3H, H-3, H-10, H-11), 5.37 5.39 (m, 1H, H-19), $5.01 \sim 5.04$ (m, 1H, H-15), 4.92 (t, $J=9.5 \mathrm{~Hz}, 1 \mathrm{H}, \mathrm{H}-4$ '), $4.88 \sim 4.90\left(\mathrm{~m}, 1 \mathrm{H}, \mathrm{H}-1^{\prime}\right), 4.66 \sim 4.69(\mathrm{~m}, 2 \mathrm{H}, \mathrm{H}-8 \mathrm{a}), 4.57$ $(\mathrm{s}, 1 \mathrm{H}, 7-\mathrm{OH}), 4.06 \sim 4.09\left(\mathrm{~m}, 1 \mathrm{H}, \mathrm{H}-5{ }^{\prime}\right), 4.00(\mathrm{~s}, 3 \mathrm{H}$, $\left.\mathrm{NOCH}_{3}\right), 3.98 \sim 4.00(\mathrm{~m}, 2 \mathrm{H}, \mathrm{H}-6, \mathrm{H}-13), 3.67 \sim 3.84(\mathrm{~m}$, 2H, H-17, H-3'), 3.43 (s, 3H, 3'- $\left.\mathrm{OCH}_{3}\right), 3.38$ (t, $J=2.5 \mathrm{~Hz}$, $1 \mathrm{H}, \mathrm{H}-2), 3.23 \sim 3.25(\mathrm{~m}, 1 \mathrm{H}, \mathrm{H}-25), 2.62\left(\mathrm{~s}, 3 \mathrm{H}, \mathrm{Ar}-\mathrm{CH}_{3}\right)$, 2.54 (m, 1H, H-12), $2.28 \sim 2.38$ (m, 5H, H-16, H-2', H-24),
$1.74 \sim 2.00\left(\mathrm{~m}, 5 \mathrm{H}, 4-\mathrm{CH}_{3}, \mathrm{H}-18\right), 1.39 \sim 1.68(\mathrm{~m}, 12 \mathrm{H}$, $\left.14-\mathrm{CH}_{3}, \mathrm{H}-20, \mathrm{H}-26, \mathrm{H}-27, \mathrm{H}-22, \mathrm{H}-23\right), 1.18 \sim 1.22(\mathrm{~m}$, $\left.6 \mathrm{H}, 5^{\prime}-\mathrm{CH}_{3}, 12-\mathrm{CH}_{3}\right), 0.79 \sim 0.96\left(\mathrm{~m}, 9 \mathrm{H}, 24,26,28-\mathrm{CH}_{3}\right)$. HRMS (ESI) calcd for $\mathrm{C}_{50} \mathrm{H}_{69} \mathrm{NO}_{12} \mathrm{Na}(\mathrm{M}+\mathrm{Na})^{+}$ 898.4718, found 898.4720.

5-甲氧亚氨基-4'-氟氯烟酸酰基弥拜霉素类似物 (5Id): 产率 27.1\%. 无色油状物. ${ }^{1} \mathrm{H}$ NMR $\left(\mathrm{CDCl}_{3}, 500\right.$ $\mathrm{MHz}) \delta: 7.97$ (s, 1H, Py-H), 5.95 5.96 (d, $J=14.8 \mathrm{~Hz}$, $1 \mathrm{H}, \mathrm{H}-9), 5.73 \sim 5.86$ (m, 2H, H-10, H-11, H-3), 5.42 $5.46(\mathrm{~m}, 1 \mathrm{H}, \mathrm{H}-19), 5.01 \sim 5.06(\mathrm{~m}, 1 \mathrm{H}, \mathrm{H}-15), 4.87 \sim 4.95$ $\left(\mathrm{m}, 2 \mathrm{H}, \mathrm{H}-1^{\prime}, \mathrm{H}-4\right.$ '), $4.74 \sim 4.76(\mathrm{~m}, 2 \mathrm{H}, \mathrm{H}-8 \mathrm{a}), 4.06 \sim 4.08$ (m, 1H, H-5'), 4.00 (s, 3H, $\left.\mathrm{NOCH}_{3}\right), 3.92$ (s, 1H, H-6), $3.87 \sim 3.89(\mathrm{~m}, 1 \mathrm{H}, \mathrm{H}-13), 3.71 \sim 3.86$ (m, 2H, H-17, H-3'), 3.58 (t, $J=2.5 \mathrm{~Hz}, 1 \mathrm{H}, \mathrm{H}-2), 3.42$ (s, 3H, 3'- $\mathrm{OCH}_{3}$ ), $3.22 \sim 3.25(\mathrm{~m}, 1 \mathrm{H}, \mathrm{H}-25), 2.56 \sim 2.58(\mathrm{~m}, 1 \mathrm{H}, \mathrm{H}-12)$, $2.28 \sim 2.34\left(\mathrm{~m}, 5 \mathrm{H}, \mathrm{H}-16, \mathrm{H}-2{ }^{\prime}, \mathrm{H}-24\right), 1.89 \sim 2.05(\mathrm{~m}, 5 \mathrm{H}$, 4- $\left.\mathrm{CH}_{3}, \mathrm{H}-18\right), 1.41 \sim 1.79\left(\mathrm{~m}, 12 \mathrm{H}, 14-\mathrm{CH}_{3}, \mathrm{H}-20, \mathrm{H}-26\right.$, H-27, H-22, H-23), $1.22 \sim 1.41\left(\mathrm{~m}, 6 \mathrm{H}, 5\right.$ ', 12- $\left.\mathrm{CH}_{3}\right), 0.79 \sim$ $0.97\left(\mathrm{~m}, 9 \mathrm{H}, 24,26,28-\mathrm{CH}_{3}\right)$. HRMS (ESI) calcd for $\mathrm{C}_{48} \mathrm{H}_{63} \mathrm{Cl}_{2} \mathrm{FN}_{2} \mathrm{O}_{12} \mathrm{Na} \quad(\mathrm{M}+\mathrm{Na})^{+}$971.3640, found 971.3644 .

5-甲氧亚氨基-13-邻氯苯甲酰基弥拜霉素类似物 (5IIa): 产率 42.3\%. 无色油状物. ${ }^{1} \mathrm{H} \mathrm{NMR}\left(\mathrm{CDCl}_{3}, 500\right.$ $\mathrm{MHz}) \delta: 7.90 \sim 7.93(\mathrm{~m}, 1 \mathrm{H}, \mathrm{H}-6-\mathrm{Ar}), 7.47 \sim 7.51(\mathrm{~m}, 2 \mathrm{H}$, $\mathrm{H}-3,4-\mathrm{Ar}), 7.38 \sim 7.40(\mathrm{~m}, 1 \mathrm{H}, \mathrm{H}-5-\mathrm{Ar}), 5.91 \sim 5.61(\mathrm{~d}$, $J=15.3 \mathrm{~Hz}, 1 \mathrm{H}, \mathrm{H}-9), 5.78 \sim 5.87$ (m, 3H, H-3, H-10, $\mathrm{H}-11), 5.41$ (s, 1H, H-13), $5.34 \sim 5.37$ (m, 1H, H-19), $5.14 \sim 5.16(\mathrm{~m}, 1 \mathrm{H}, \mathrm{H}-15), 4.70 \sim 4.73(\mathrm{~m}, 2 \mathrm{H}, \mathrm{H}-8 \mathrm{a}), 4.57$ (s, 1H, 7-OH), 4.00 (s, 3H, = $\mathrm{NOCH}_{3}$ ), 3.89 (s, 1H, H-6), $3.57 \sim 3.59$ (m, 1H, H-17), 3.38 (dd, $J=2.0,4.5 \mathrm{~Hz}, 1 \mathrm{H}$, $\mathrm{H}-2), 3.13$ (d, $J=8.0 \mathrm{~Hz}, 1 \mathrm{H}, \mathrm{H}-25), 2.75 \sim 2.78(\mathrm{~m}, 1 \mathrm{H}$, $\mathrm{H}-12), 2.28 \sim 2.30(\mathrm{~m}, 2 \mathrm{H}, \mathrm{H}-16), 1.77 \sim 2.29(\mathrm{~m}, 6 \mathrm{H}$, $\left.\mathrm{H}-24,4-\mathrm{CH}_{3}, \mathrm{H}-18\right), 1.35 \sim 1.70\left(\mathrm{~m}, 12 \mathrm{H}, 14-\mathrm{CH}_{3}, \mathrm{H}-20\right.$, $\mathrm{H}-26, \mathrm{H}-27, \mathrm{H}-22, \mathrm{H}-23), 1.16 \sim 1.18\left(\mathrm{~m}, 3 \mathrm{H}, 12-\mathrm{CH}_{3}\right)$, $0.77 \sim 0.86\left(\mathrm{~m}, 9 \mathrm{H}, 24,26,28-\mathrm{CH}_{3}\right)$. HRMS (ESI) calcd for $\mathrm{C}_{42} \mathrm{H}_{54} \mathrm{ClNO}_{9} \mathrm{Na}(\mathrm{M}+\mathrm{Na})^{+} 774.3385$, found 774.3389 .

5-甲氧亚氨基-13-对甲基苯甲酰基弥拜霉素类似物 (5IIb): 产率 45.6\%. 无色油状物. ${ }^{1} \mathrm{H} \mathrm{NMR}\left(\mathrm{CDCl}_{3}, 500\right.$ $\mathrm{MHz}) \delta: 8.01(\mathrm{~m}, 2 \mathrm{H}, \mathrm{H}-2,6-\mathrm{Ar}), 7.28 \sim 7.30(\mathrm{~m}, 2 \mathrm{H}$, Ar-H), $5.92 \sim 5.94$ (m, 1H, H-9), $5.77 \sim 5.89$ (m, 3H, H-3, $\mathrm{H}-10, \mathrm{H}-11), 5.38$ (s, 1H, H-13), 5.34 $5.36(\mathrm{~m}, 1 \mathrm{H}$, $\mathrm{H}-19), 5.10 \sim 5.12(\mathrm{~m}, 1 \mathrm{H}, \mathrm{H}-15), 4.67 \sim 4.69(\mathrm{~m}, 2 \mathrm{H}$, $\mathrm{H}-8 \mathrm{a}), 4.56$ (s, 1H, 7-OH), $4.00\left(\mathrm{~s}, 3 \mathrm{H},=\mathrm{NOCH}_{3}\right), 3.87$ (s, 1H, H-6), $3.58 \sim 3.60$ (m, 1H, H-17), 3.38 (dd, $J=2.0$, $4.5 \mathrm{~Hz}, 1 \mathrm{H}, \mathrm{H}-2), 3.12$ (d, $J=8.0 \mathrm{~Hz}, 1 \mathrm{H}, \mathrm{H}-25), 2.76 \sim$ 
$2.78(\mathrm{~m}, 1 \mathrm{H}, \mathrm{H}-12), 2.64\left(\mathrm{~s}, 3 \mathrm{H}, \mathrm{Ar}-\mathrm{CH}_{3}\right), 2.24 \sim 2.26(\mathrm{~m}$, $2 \mathrm{H}, \mathrm{H}-16), 1.77 \sim 2.27$ (m, 6H, 4,24- $\left.\mathrm{CH}_{3}, \mathrm{H}-18\right), 1.26 \sim$ 1.65 (m, 12H, 14- $\left.\mathrm{CH}_{3}, \mathrm{H}-20, \mathrm{H}-26, \mathrm{H}-27, \mathrm{H}-22, \mathrm{H}-23\right)$, $1.12 \sim 1.14\left(\mathrm{~m}, 3 \mathrm{H}, 12-\mathrm{CH}_{3}\right), 0.76 \sim 0.89(\mathrm{~m}, 9 \mathrm{H}$, 24,26,28- $\mathrm{CH}_{3}$ ). HRMS (ESI) calcd for $\mathrm{C}_{43} \mathrm{H}_{57} \mathrm{NO}_{9} \mathrm{Na}(\mathrm{M}+$ $\mathrm{Na})^{+}$754.3931, found 754.3936 .

5-甲氧亚氨基-13-邻甲基苯甲酰基弥拜霉素类似物 (5IIc): 产率 55.7\%. 无色油状物. ${ }^{1} \mathrm{H}$ NMR $\left(\mathrm{CDCl}_{3}, 500\right.$ $\mathrm{MHz}) \delta: 8.02(\mathrm{~m}, 1 \mathrm{H}, \mathrm{H}-6-\mathrm{Ar}), 7.44 \sim 7.46(\mathrm{~m}, 1 \mathrm{H}$, H-4-Ar), $7.29 \sim 7.31(\mathrm{~m}, 2 \mathrm{H}, \mathrm{H}-3,5-\mathrm{Ar}), 5.93 \sim 5.95(\mathrm{~m}$, 1H, H-9), $5.78 \sim 5.88$ (m, 3H, H-3, H-10, H-11), 5.39 (s, $1 \mathrm{H}, \mathrm{H}-13), 5.34 \sim 5.36(\mathrm{~m}, 1 \mathrm{H}, \mathrm{H}-19), 5.10 \sim 5.13(\mathrm{~m}, 1 \mathrm{H}$, H-15), $4.68 \sim 4.70$ (m, 2H, H-8a), 4.58 (s, 1H, 7-OH), 4.00 $\left(\mathrm{s}, 3 \mathrm{H},=\mathrm{NOCH}_{3}\right), 3.88(\mathrm{~s}, 1 \mathrm{H}, \mathrm{H}-6), 3.58 \sim 3.61(\mathrm{~m}, 1 \mathrm{H}$, H-17), 3.39 (dd, $J=2.0,4.5 \mathrm{~Hz}, 1 \mathrm{H}, \mathrm{H}-2), 3.12$ (d, $J=8.0$ $\mathrm{Hz}, 1 \mathrm{H}, \mathrm{H}-25), 2.75 \sim 2.78(\mathrm{~m}, 1 \mathrm{H}, \mathrm{H}-12), 2.64(\mathrm{~s}, 3 \mathrm{H}$, $\left.\mathrm{Ar}-\mathrm{CH}_{3}\right), \quad 2.25 \sim 2.27(\mathrm{~m}, 2 \mathrm{H}, \mathrm{H}-16), 1.76 \sim 2.26(\mathrm{~m}, 6 \mathrm{H}$, $\left.\mathrm{H}-24,4-\mathrm{CH}_{3}, \mathrm{H}-18\right), 1.26 \sim 1.65\left(\mathrm{~m}, 12 \mathrm{H}, 14-\mathrm{CH}_{3}, \mathrm{H}-20\right.$, $\mathrm{H}-26, \mathrm{H}-27, \mathrm{H}-22, \mathrm{H}-23), 1.13 \sim 1.16\left(\mathrm{~m}, 3 \mathrm{H}, 12-\mathrm{CH}_{3}\right)$, $0.75 \sim 0.86\left(\mathrm{~m}, 9 \mathrm{H}, 24,26,28-\mathrm{CH}_{3}\right)$. HRMS (ESI) calcd for $\mathrm{C}_{43} \mathrm{H}_{57} \mathrm{NO}_{9} \mathrm{Na}(\mathrm{M}+\mathrm{Na})^{+} 754.3931$, found 754.3930 . 5-甲氧亚氨基-13-氟氯吡啶甲酰基弥拜霉素类似物
(5IIe): 产率 35.8\%. 无色油状物. ${ }^{1} \mathrm{H}$ NMR $\left(\mathrm{CDCl}_{3}, 500\right.$ MHz) $\delta: 8.05$ (s, 1H, Py-H), 5.71 5.95 (m, 3H, H-9, H-3, $\mathrm{H}-10, \mathrm{H}-11), 5.43$ (s, 1H, H-13), $5.35 \sim 5.37(\mathrm{~m}, 1 \mathrm{H}$, $\mathrm{H}-19), 5.06 \sim 5.08(\mathrm{~m}, 1 \mathrm{H}, \mathrm{H}-15), 4.68 \sim 4.70(\mathrm{~m}, 2 \mathrm{H}$, $\mathrm{H}-8 \mathrm{a}), 4.57$ (s, 1H, 7-OH), $4.00\left(\mathrm{~s}, 3 \mathrm{H},=\mathrm{NOCH}_{3}\right), 3.89$ (s, 1H, H-6), $3.62 \sim 3.65$ (m, 1H, H-17), 3.38 (dd, $J=2.0$, $4.5 \mathrm{~Hz}, 1 \mathrm{H}, \mathrm{H}-2), 3.15$ (d, $J=8.0 \mathrm{~Hz}, 1 \mathrm{H}, \mathrm{H}-25), 2.79$ 2.83 (m, 1H, H-12), $2.27 \sim 2.29$ (m, 2H, H-16), 1.73 2.04 $\left(\mathrm{m}, 6 \mathrm{H}, 24,4-\mathrm{CH}_{3}, \mathrm{H}-18\right), 1.35 \sim 1.66\left(\mathrm{~m}, 12 \mathrm{H}, 14-\mathrm{CH}_{3}\right.$, $\mathrm{H}-20, \mathrm{H}-26, \mathrm{H}-27, \mathrm{H}-22, \mathrm{H}-23), 1.14 \sim 1.16(\mathrm{~m}, 3 \mathrm{H}$, $\left.12-\mathrm{CH}_{3}\right), 0.77 \sim 0.88\left(\mathrm{~m}, 9 \mathrm{H}, 24,26,28-\mathrm{CH}_{3}\right)$. HRMS (ESI) calcd for $\mathrm{C}_{41} \mathrm{H}_{51} \mathrm{Cl}_{2} \mathrm{FN}_{2} \mathrm{O}_{9} \mathrm{Na}(\mathrm{M}+\mathrm{Na})^{+}$827.2853, found 827.2858

\section{3 生物活性测定}

测试方法为浸叶片法，测定化合物 4Ia 5IId 分别 对朱砂叶螨 (Tetranychus cinnabarinus)、南方粘虫 (Mythimna sepatara) 和虫豆蚜(Aphis fabae)这三种四龄 幼虫的室内生物活性. 供试温度为 $(25 \pm 1){ }^{\circ} \mathrm{C}$, 各化合 物的普笁浓度分别为 $0.006,200$ 和 $200 \mathrm{mg} \cdot \mathrm{L}^{-1}$, 每个测 试浓度平行 3 次. 以依维菌素作对照, 校正死亡率以清 水为对照, 按 Abbott 公式计算得出, 测定结果见表 1 和 2 所示.

表 1 所有目标化合物的杀虫活性(抑制率/\%)

Table 1 The insecticidal activities (inhibition rate/\%) of title compounds

\begin{tabular}{|c|c|c|c|c|}
\hline 化合物 & $\mathrm{R}^{1}$ or $\mathrm{R}^{2}$ & 朱砂叶螨 $\left(0.006 \mathrm{mg} \cdot \mathrm{L}^{-1}\right)^{a}$ & 南方粘虫 $\left(200 \mathrm{mg} \cdot \mathrm{L}^{-1}\right)^{a}$ & 虫豆蚜 $\left(200 \mathrm{mg} \cdot \mathrm{L}^{-1}\right)^{a}$ \\
\hline 依维菌素 & . & 57.9 & 100 & 100 \\
\hline $4 \mathrm{Ia}$ & & 36.2 & 89.6 & 76.1 \\
\hline $4 \mathrm{IIa}$ & & 11.8 & 100 & 100 \\
\hline $4 \mathrm{Ib}$ & & 19.5 & 90.5 & 68.6 \\
\hline $4 \mathrm{IIb}$ & & 16.4 & 100 & 100 \\
\hline 4Ic & & 24.5 & 88.9 & 61.6 \\
\hline 4IIIc & & 23.5 & 95.2 & 100 \\
\hline 4Id & & 14.6 & 70.5 & 87.2 \\
\hline 4IId & & 15.2 & 88.5 & 98.2 \\
\hline $5 \mathrm{Ia}$ & & 18.3 & 0 & 7.9 \\
\hline $5 \mathrm{IIa}$ & & 9.1 & 0 & 11.2 \\
\hline $5 \mathrm{Ib}$ & & 15.5 & 0 & 0 \\
\hline $5 \mathrm{IIb}$ & & 6.3 & 0 & 12.4 \\
\hline 5Ic & & 6.7 & 0 & 2.3 \\
\hline $5 \mathrm{IIc}$ & & 5.2 & 0 & 22.1 \\
\hline
\end{tabular}




\begin{tabular}{ccccc}
\hline 化合物 & $\mathrm{R}^{1}$ or $\mathrm{R}^{2}$ & 朱砂叶螨 $\left(0.006 \mathrm{mg} \cdot \mathrm{L}^{-1}\right)^{a}$ & 南方粘虫 $\left(200 \mathrm{mg} \cdot \mathrm{L}^{-1}\right)^{a}$ & 蚎豆蜴 $\left(200 \mathrm{mg} \cdot \mathrm{L}^{-1}\right)^{a}$ \\
\hline 5Id & & 5.3 & 0 & 0 \\
5IId & & 6.3 & 0 & 8.9 \\
\hline
\end{tabular}

测试浓度.

表 2 化合物 4IIa, 4IIb 和依维菌素分别对南方粘虫和虫豆蚜的 $\mathrm{LC}_{50}$ 值

Table 2 The larvicidal $\mathrm{LC}_{50}$ values of compounds $4 \mathbf{I I a}$, 4IIb and ivermectin against Mythimna sepatara and Aphis fabae

\begin{tabular}{|c|c|c|c|c|c|c|}
\hline \multirow{2}{*}{ 化合物 } & \multicolumn{3}{|c|}{ 南方粘虫 } & \multicolumn{3}{|c|}{ 贵豆蚜 } \\
\hline & $\mathrm{LC}_{50} /\left(\mathrm{mg} \cdot \mathrm{L}^{-1}\right)$ & 测试浓度/(mg・L $\left.{ }^{-1}\right)$ & 死亡率/\% & $\mathrm{LC}_{50} /\left(\mathrm{mg} \cdot \mathrm{L}^{-1}\right)$ & 测试浓度 $/\left(\mathrm{mg} \cdot \mathrm{L}^{-1}\right)$ & 死亡率 $/ \%$ \\
\hline \multirow{6}{*}{$4 \mathrm{III}$} & \multirow{6}{*}{0.15} & 1 & 100.0 & \multirow{6}{*}{0.31} & 2 & 100.0 \\
\hline & & 0.5 & 89.3 & & 1 & 86.2 \\
\hline & & 0.25 & 70.1 & & 0.5 & 75.0 \\
\hline & & 0.125 & 45.6 & & 0.25 & 45.8 \\
\hline & & 0.0625 & 25.9 & & 0.125 & 10.3 \\
\hline & & 0.03125 & 0 & & 0.0625 & 0 \\
\hline \multirow{6}{*}{$4 \mathrm{IIJ}$} & \multirow{6}{*}{0.29} & 1 & 94.5 & \multirow{6}{*}{0.19} & 2 & 100.0 \\
\hline & & 0.5 & 70.6 & & 1 & 90.4 \\
\hline & & 0.25 & 43.1 & & 0.5 & 78.2 \\
\hline & & 0.125 & 22.3 & & 0.25 & 57.6 \\
\hline & & 0.0625 & 10.2 & & 0.125 & 38.5 \\
\hline & & 0.03125 & 0 & & 0.0625 & 21.0 \\
\hline \multirow{6}{*}{ 依维菌素 } & \multirow{6}{*}{0.19} & 1 & 100.0 & \multirow{6}{*}{0.05} & 0.5 & 91.0 \\
\hline & & 0.5 & 90.9 & & 0.25 & 80.3 \\
\hline & & 0.25 & 66.7 & & 0.125 & 69.8 \\
\hline & & 0.125 & 29.1 & & 0.0625 & 58.6 \\
\hline & & 0.0625 & 6.4 & & 0.03125 & 43.2 \\
\hline & & 0.03125 & 0 & & 0.015625 & 25.6 \\
\hline
\end{tabular}

\section{2 结果与讨论}

\section{1 合成方法}

采用催化水解法制备单糖弥拜霉素类似物, 溶剂、 反应温度、时间和酸浓度都会对反应有很大影响. 由于 反应易同时失去两个糖而生成无糖弥拜霉素类似物, 故 反应需选用极性较低的异丙醇作溶剂, 在冰水浴且酸浓 度小于 $3 \%$, 反应时间小于 $18 \mathrm{~h}$ 的条件下催化水解制得 单糖弥拜霉素类似物. 由于异丙醇的大量存在后处理时 易发生乳化现象, 可向提取液中加入适量 $\mathrm{NaCl}$ 颗粒从 而有效控制乳化现象. 无糖弥拜需素类似物的制备方法 和操作与单糖弥拜霉素类似物基本相似, 只是选用极性 较强的甲醇作为溶剂, 延长反应时间, 增大酸的反应浓 度. 另外 5-O-TBDMS-单糖弥拜霉素类似物 3I 制备中选 用 TBDMS-Cl 作为保护基团, 为了提高收率, 使底物反 应充分, TBDMS-Cl 应适当过量, 单糖弥拜雱素类似物 与 TBDMS-Cl 的物质的量为 $1: 2.2$. 单糖弥拜霉素类似 物中有两个羟基, 反应时 $\mathrm{C}-5-\mathrm{OH}$ 的活性要高于 C-4'-OH，但过量 TBDMS-Cl 仍会与 C-4'-OH 反应，所以 控制好反应时间(约 $6 \mathrm{~h}$ ) 是减少此副产物的关键.
5-O-TBDMS- 无糖弥拜霉素类似物 (3II) 的制备与 5-O-TBDMS-单糖弥拜霉素类似物 3I 相似. 但无糖弥拜 霉素类似物中 $\mathrm{C}-13-\mathrm{OH}$ 较单糖弥拜霉素类似物 C-4'-OH 活泼，易参与反应形成副产物，故应将反应时间控制在 $5 \mathrm{~h}$, 较为合适.

\section{2 目标化合物的杀虫活性}

采用浸叶片法, 经过普篮和复篮测定各化合物分别 对朱砂叶螨(Tetranychus cinnabarinus)、南方粘虫 (Mythimna sepatara) 和虫豆蚜 (Aphis fabae) 的杀虫活性. 由表 1 和表 2 可知, 在 $0.006 \mathrm{mg} \cdot \mathrm{L}^{-1}$ 的测试浓度下所有 目标化合物包括依维菌素在内均对朱砂叶螨表现出较 差的活性，除了依维菌素，其他的化合物均为达到 50\% 的杀虫活性. 另外在 $200 \mathrm{mg} \cdot \mathrm{L}^{-1}$ 的测试浓度下化合物 4Ia 4IId 和依维菌素对南方粘虫和蚕豆蚵均表现出较 好的杀虫活性, 尤其化合物 4IIa 和 4IIb 的杀虫活性最 高. 值得关注的是虽然化合物 $4 \mathrm{IIb}\left(\mathrm{LC}_{50}=0.29 \mathrm{mg} \cdot \mathrm{L}^{-1}\right)$ 对南方粘虫的杀虫活性较依维菌素稍低, 但是化合物 $4 \mathrm{IIa}\left(\mathrm{LC}_{50}=0.15 \mathrm{mg} \cdot \mathrm{L}^{-1}\right)$ 对南方粘虫的杀虫活性比依维 菌素 $\left(\mathrm{LC}_{50}=0.19 \mathrm{mg} \cdot \mathrm{L}^{-1}\right)$ 要高. 化合物 4 IIId 在 $200 \mathrm{mg} \cdot$ $\mathrm{L}^{-1}$ 试验浓度下对南方粘虫和䖯豆蚜也表现出良好的杀 
虫活性, 死亡率分别为 $88.5 \%$ 和 $98.2 \%$. 从表 1 可知, 单 糖化合物(4Ia 4II) 活性普遍比无糖基的低, 基本上未 达到 90\%. 当 5-位的羟基转化为甲氧肜之后活性大大降 低，甚至无杀虫活性.

\section{3 结论}

以弥拜霉素类似物依维菌素为原料, 利用不同溶 剂、反应时间和酸的浓度对依维菌素分别脱去一个和两 个糖基, 然后与相应的酰氯进行酯化, 再肜化制得收率 较好的两个系列弥拜霉素类似物化合物 4Ia $~ 5 I I d$. 以 无糖基系列的弥拜霉素类似物 4IIa 5IId 为目标化合 物, 单糖基系列的弥拜霉素类似物 $4 \mathrm{II} \sim 5 \mathrm{Id}$ 作比较, 分 别对朱砂叶螨(Tetranychus cinnabarinus)、南方粘虫 (Mythimna sepatara) 和虽豆蚜(Aphis fabae)进行室内杀 虫活性测定. 生测结果表明, 无糖基系列的弥拜霉素类 似物 4III $\sim 5 I I d$ 对南方粘虫和蚕豆蚜的杀虫活性比单 糖基系列的弥拜霉素类似物 4Ia $\sim 5$ Id 高很多, 尤其是 13 位上连接邻氯苯甲酰基(4IIa)和邻甲氧基苯甲酰基 (4IIb)的两个化合物, 具备发展高活性、低成本的新型弥 拜需素类杀虫、驱虫剂的潜力, 有进一步深入研究的价
值.

致谢 湖南化工研究院对所有目标化合物进行生物活 性试验.

\section{References}

[1] Davies, H. G.; Green, R. H. Chem. Soc. Rev. 1991, 20, 211.

[2] Fisher, M. H.; Mrozik, H. In Ivermectin and Abamectin, Ed.: Campbell, W. C., Springer, New York, 1989, p. 1.

[3] Putter, I.; Martinsville, N. J. US 4144352, 1979 [Chem. Abstr. 1979, 63, 104541].

[4] Takeshiba, H.; Sato, K.; Yanai, T.; Yokoi, S.; Ichinose, R. JP 09143183, 1997 [Chem. Abstr. 1997, 127, 81288].

[5] Williams, D. R.; Barner, B. A.; Nishitani, K.; Phillips, J. G. J. Am. Chem. Soc. 1982, 104, 4708.

[6] Liao, L. A.; Guo, Q. Z. Chin. J. Synth. Chem.. 1995, 3, 215 (in Chinese).

(廖联安, 郭奇珍, 合成化学, 1995, 3, 215.)

[7] Sato, K.; Saito, A.; Toyama, T. EP 765879, 1997 [Chem. Abstr. 1997, 126, 263962].

[8] Zhao, J. H.; Xu, X. J.; Ji, M. H.; Cheng, J. L.; Zhu, G. N. J. Agric. Food Chem. 2011, 59, 4836

[9] Zhao, J. H.; Ji, M. H.; Xu, X. H; Cheng, J. L.; Zhu, G. N. Chin. Chem. Lett. 2010, 21, 1391.

[10] Coutrot, P.; Ghribi, A. Synthesis 1986, 661. 